\title{
TROPOMI, the solar backscatter satellite instrument for air quality and climate, heads towards detailed design
}

\author{
Johan de Vries ${ }^{* a}$, Robert Voors ${ }^{\mathrm{a}}, \mathrm{A}^{\prime}$ gnes Mika ${ }^{\mathrm{b}}$, Gerard Otter ${ }^{\mathrm{c}}$, Nick van der Valk ${ }^{\mathrm{c}}$, Ilse Aben ${ }^{\mathrm{d}}$, Ruud \\ Hoogeveen $^{\mathrm{d}}$, Annemieke Gloudemans ${ }^{\mathrm{d}}$, Marcel Dobber ${ }^{\mathrm{e}}$, Pepijn Veefkind ${ }^{\mathrm{e}}$, Pieternel Levelt ${ }^{\mathrm{e}}$ \\ ${ }^{a}$ Dutch Space B.V. Mendelweg 30 - 2333 CS Leiden, The Netherlands \\ ${ }^{\mathrm{b}}$ BMT ARGOSS, Voorsterweg 28, 8316 PT Marknesse, The Netherlands \\ ${ }^{\mathrm{c}}$ TNO Netherlands Organization for Applied Technological Research, Stieltjesweg 1, 2628 CK Delft \\ ${ }^{\mathrm{d}}$ SRON Netherlands Institute for Space Research, Sorbonnelaan 23584 CA Utrecht, The \\ Netherlands \\ ${ }^{\mathrm{e}}$ KNMI Royal Netherlands Meteorological Institute, Wilhelminalaan 10, 3732 GK De Bilt
}

\begin{abstract}
The Tropospheric Monitoring Instrument (TROPOMI) is currently planned for launch on ESA's Sentinel 5 precursor satellite in the time frame of 2014. TROPOMI is an ultraviolet-to-SWIR wavelengths imaging spectrograph that uses two-dimensional detectors to register both the spectrum and the swath perpendicular to the flight direction. The swath is about 110 degrees wide to allow daily global coverage from the polar orbit of the Sentinel 5 precursor satellite. The instrument follows the heritage of SCIAMACHY (ENVISAT, launch 2002) and OMI (AURA, launch 2004), but it has been improved in several ways: the ground resolution is down to $7 \times 7 \mathrm{~km}^{2}$, the instrument is fit for low albedo scenes and the wavelength bands are optimized using the SCIAMACHY and OMI heritages to have the best trace gas products. The first two improvements basically mean that the instrument aperture is much larger for TROPOMI and, related to this, the reading of the detectors much faster. The selected wavelength bands for TROPOMI are UV1 (270-310 nm), UV2 $(310-370 \mathrm{~nm})$, VIS $(370-500 \mathrm{~nm})$, NIR $(675-775 \mathrm{~nm})$ and SWIR $(2305-2385 \mathrm{~nm})$. The first three bands are very similar to the OMI bands, the NIR has been added to improve on clouds and air mass corrections and the SWIR allows measuring $\mathrm{CH}_{4}$ and $\mathrm{CO}$. The paper discusses the development status on several topics, such as detector selection and polarization scrambler performance simulations using the TIDE grid based level 2 scene simulator.
\end{abstract}

Keywords: atmospheric remote sensing, UV-SWIR spectrograph, hyperspectral imaging

\section{PROGRAM STATUS}

The Sentinel-5 precursor mission has been agreed by the ESA ministerial conference in November last year for launch in 2014. The TROPOMI instrument is split into separate modules for the UV-NIR and SWIR wavelength parts where the SWIR part together with the spacecraft is supplied via ESA and the UV-NIR part is a Dutch national contribution as was formalized by the Dutch minister of Economic affairs on July $6^{\text {th }}$.

At this moment there are in parallel the Dutch national phase B for the UV-NIR and the industrial selection process for the SWIR and the spacecraft.

In the Netherlands the main partners are Dutch Space, TNO, SRON, KNMI and BMT ARGOSS. Dutch Space acts as prime industry for the complete TROPOMI instrument. TNO is responsible for the optical design (in the Dutch national phase both the UVNIR and SWIR) and KNMI and SRON have the scientific lead for the UV-NIR and the SWIR respectively. BMT ARGOSS is preparing the commercial use of the TROPOMI data.

ESA is preparing selection of the satellite prime contractor and, together with Dutch Space, the contractors for the SWIR and electronics modules.

”i.de.vries@dutchspace.nl; phone +31 71 5245879; http://www.dutchspace.nl

Sensors, Systems, and Next-Generation Satellites XIII, edited by Roland Meynart,

Steven P. Neeck, Haruhisa Shimoda, Proc. of SPIE Vol. 7474, 747409 • ( 2009

SPIE · CCC code: $0277-786$ X/09/\$18 $\cdot$ doi: $10.1117 / 12.830087$

Proc. of SPIE Vol. 7474 747409-1 


\section{INSTRUMENT CONCEPT}

TROPOMI builds upon the heritages of the SCIAMACHY ${ }^{1}$ and the $\mathrm{OMI}^{2}$ instruments. It will be launched in a sun synchronous polar orbit.

SCIAMACHY, launched in 2002 on ESA's ENVISAT satellite, is a scanning spectrograph with very flexible viewing possibilities (a.o. nadir and limb), a wide wavelength range $(240-2400 \mathrm{~nm})$ and daily coverage of the Earth in 6 days. The scanning concept of SCIAMACHY results in ground pixels of $30 \times 120 \mathrm{~km}^{2}$, depending on the instrument mode. SCIAMACHY measures the globe in 6 days.

OMI was launched on NASA's AURA satellite in 2004 and is a staring spectrograph, using two-dimensional CCD detectors to register wavelength and across-flight swath. OMI is dedicated to nadir observations and also limits its wavelength range from 270 to $500 \mathrm{~nm}$. This wavelength range has the absorption bands of most of the important trace gases but not all. Fig. 1 shows the gases absorbing in this wavelength range.

Although the staring concept of OMI has a smaller telescope aperture as compared to the SCIAMACHY scanning concept, the staring concept allows for much better spatial resolution because it measures all ground pixels in the swath simultaneously instead of sequentially: the OMI has nominally $13 \times 24 \mathrm{~km}^{2}$ pixels and has a very wide swath width (114 degrees), sufficient to measure the globe in a single day.

Because of the different concepts, SCIAMACHY and OMI have also very different approaches towards polarization. SCIAMACHY has a polarization sensitivity depending on the scan angle and has dedicated Polarization Measurement Devices (PMD's) to measure polarization, be-it in reduced spectral resolution as compared to the main spectrograph channels. It has the advantage of having polarization data available to improve on e.g. aerosol and cloud characterization but the disadvantage of added complexity in both the instrument hardware and the calibration. The latter is important because SCIAMACHY has two scanner mirrors to accommodate the nadir and limb measurement modes.

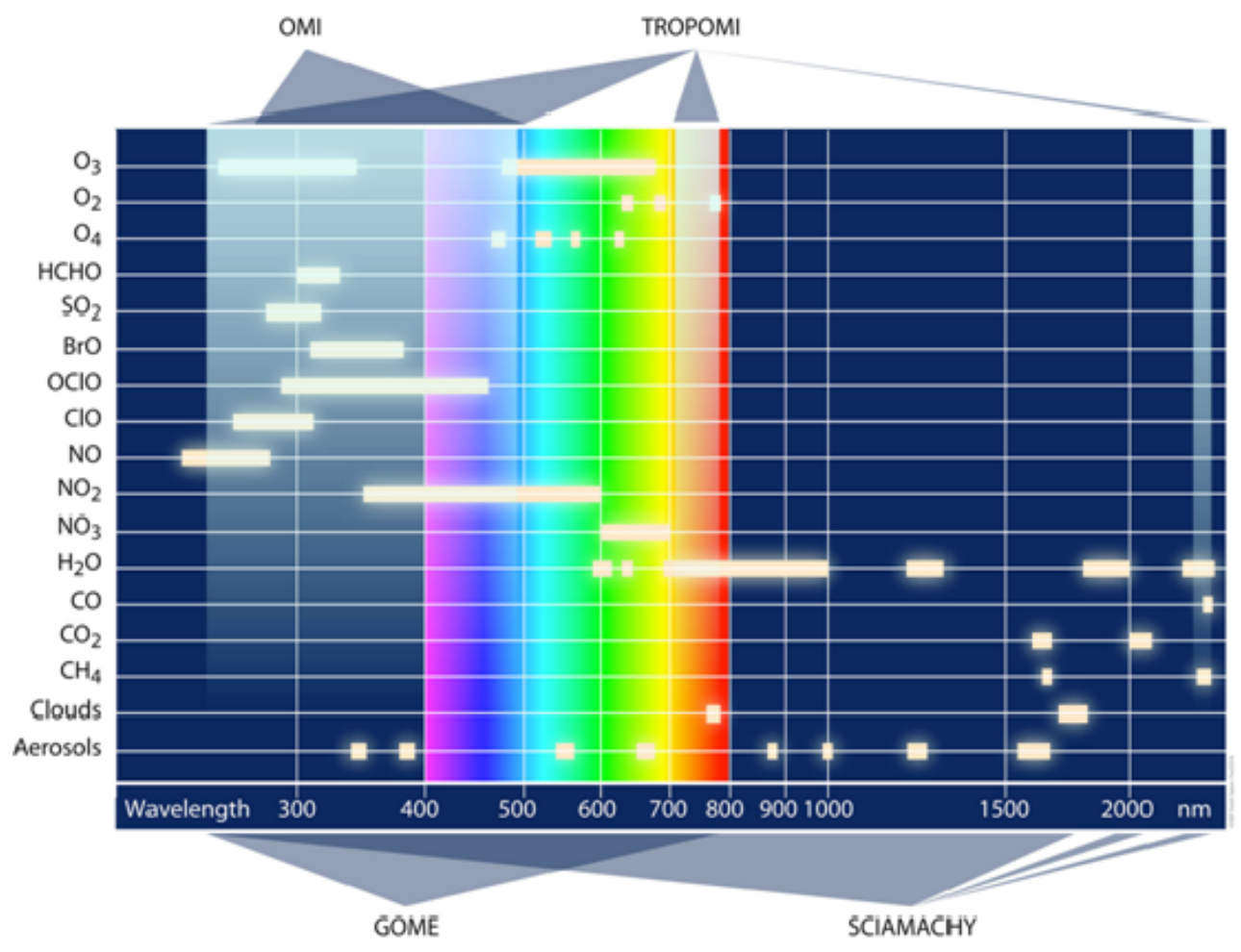

Fig. 1 Wavelength ranges of the important UV-SWIR trace gas instruments, together with the absorbing gases 
The OMI telescope has a central volume where all rays pass through and allows having a small depolarizer (polarization scrambler) in this location. Although lacking polarization information in this way, the OMI hardware, radiometric calibration and correction software are much more straightforward.

UV-SWIR trace gas spectroscopy from space was originally primarily used for climate research (e.g. the ozone layer observations are very important) but it also has a high sensitivity to boundary layer trace gases. This makes the technique suitable to measure air quality from space next to climate parameters. OMI's daily coverage and fine spatial resolution meant a significant step forward in observing air quality from space and TROPOMI is meant to make the next order of magnitude improvement for these measurements.

TROPOMI has the following heritage properties.

- It uses OMI's staring concept to have small ground pixels in combination with daily global coverage and polarization insensitivity

- $\quad$ TROPOMI basically has the UV-VIS spectral channels from OMI, i.e. a UV1 (270 -308 nm), UV2 (308 - 370 $\mathrm{nm})$, VIS (370 - $490 \mathrm{~nm})$

- It adds an $\mathrm{O}_{2} \mathrm{~A}$ band similar to SCIAMACHY for cloud detection meant to have improved air mass determinations as compared to OMI (NIR $710-775 \mathrm{~nm}$ )

- It has SCIAMACHY's channel 8 to measure $\mathrm{CH}_{4}$ and $\mathrm{CO}$ and to extend the overall wavelength range for aerosol measurements (SWIR $2305-2385 \mathrm{~nm}$ )

And TROPOMI has two important improvements compared to OMI and SCIAMACHY.

- The ground pixel size is improved to $7 \times 7 \mathrm{~km}^{2}$, this is $6 \times$ better as compared to OMI and $73 \mathrm{x}$ better than SCIAMACHY

- The instrument is designed to meet the signal-to-noise requirements for very dark scenes ( $2 \%$ albedo in the UVNIR and $5 \%$ for the SWIR): for the visible wavelengths TROPOMI is $15 \mathrm{x}$ more sensitive as compared to OMI

In short, TROPOMI will have more than the best of the two worlds of SCIAMACHY and OMI and it will form a very significant improvement for determining sources and sinks of trace gases.

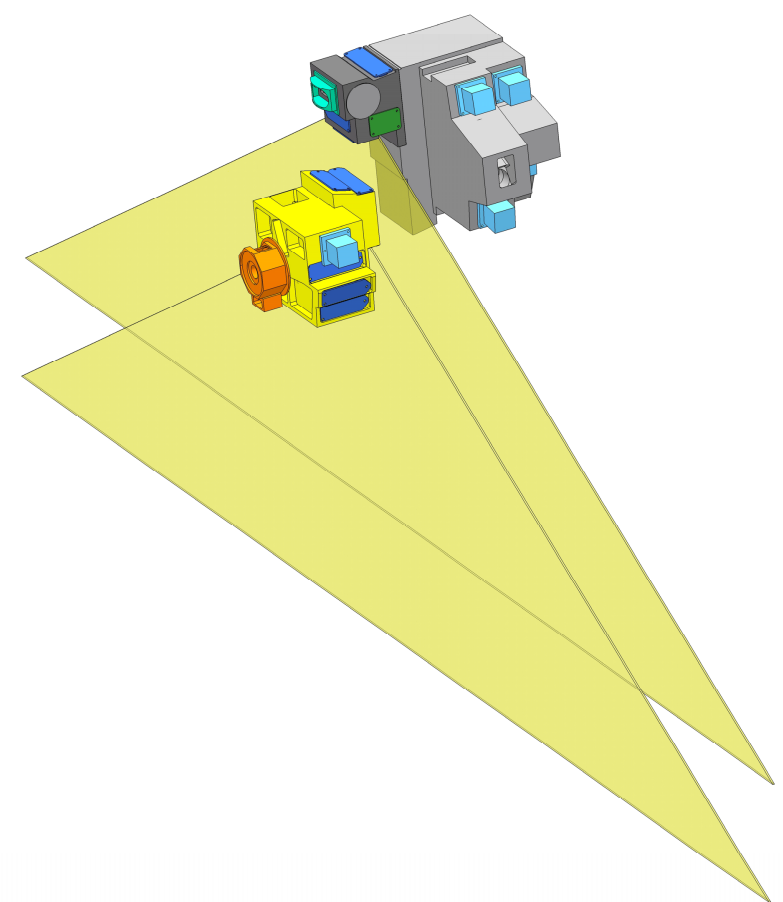

Fig.2 TROPOMI instrument layout with separate modules for the SWIR (in-front) and the UV-NIR and (in yellow) the wide Earth viewing field-of-view 


\section{DYNAMIC RANGE}

We have explained above that TROPOMI is designed to meet its signal-to-noise requirements for very dark scenes: with $2 \%$ albedo in the UV-NIR and $5 \%$ in the SWIR. This is a difficult requirement since dark scenes may be partly cloudy resulting in radiances with effective albedo of $65-100 \%$.
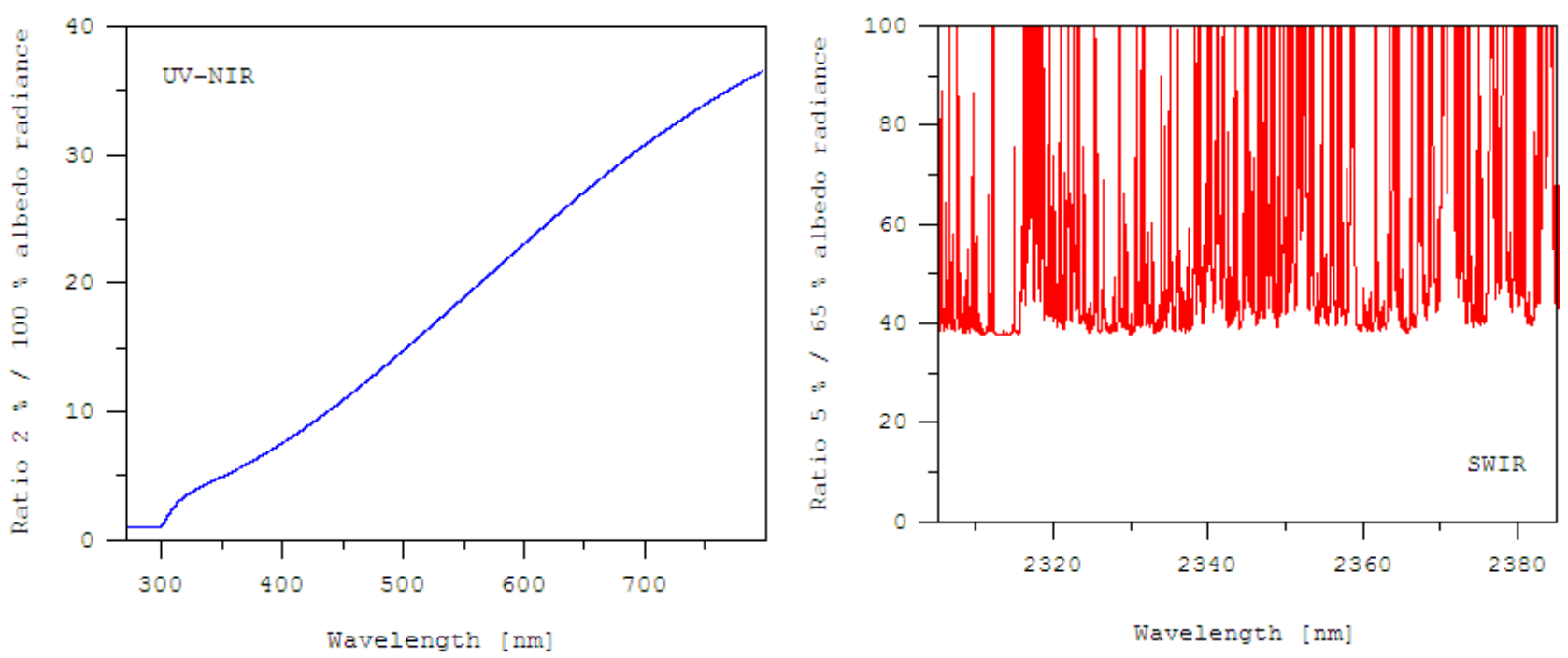

Fig.3 Ratio of maximum / minimum radiances for the UV-NIR (left graph) and SWIR (right graph, the continuum ratio 40 is most relevant, not so much the peaks), to show TROPOMI's dynamical range

The highest signal-to-noise requirement is 1500 for the VIS channel. We see that for this channel, the maximum contrast is about a factor 15 . This means that, even in the shot noise limit, the signal-to-noise requirement of 1500 for minimum signal translates to $1500 \times \sqrt{ } 15=6000$ for the maximum signal.

Also, the signal to be accumulated per measurement is $6000^{2}=3.4 \times 10^{7}$ electrons. For a pixel full well of $500 \mathrm{ke}$ and a binning factor of 4 , this means that we need $3.4 .10^{7} /\left(4 \times 5.10^{5}\right)=17$ exposures to obtain this signal level. Together with the $7 \mathrm{~km}$ satellite travel time of about $1 \mathrm{~s}$, this means that we have to read the detector in $1 / 17 \cong 50 \mathrm{~ms}$. For $1000 \times 250$ pixels this yields a pixel readout frequency of $5 \mathrm{MHz}$.

These estimates sofar have not taken into account the readout noise but we hope to have shown that TROPOMI is challenging with respect to detector and electronics design.

The SWIR channel is with respect to pixel readout frequency not as demanding as the VIS channel, primarily because the required signal-to-noise is lower $(100-120)$.

\section{DETECTOR SELECTION}

In TROPOMI we have per detector channel about 1000 spectral x 220 swath viewing pixels. However, there are a few arguments to bin detector pixels in the swath direction and therefore we require the detector to be larger $(\sim 1000 \mathrm{x} 1000$ pixels). The arguments are the following.

- It allows to program the number of pixels to be binned and increases therefore the flexibility of the instrument

- Per ground pixel, the effective pixel full well is larger and allows accumulating more electrons per exposure

- It avoids using cylindrical elements in the detector proximity optics

The number of pixels binned is 4 in the centre of the swath and decreases to 2 in the extremes of the swath, to compensate partly for the increase of the ground pixel size with increasing swath angle. 
There are a lot more properties to be required from these detectors, as there are.

- Good quantum efficiency for all wavelengths; for the UV this means we need a back-illuminated sensor, for the NIR it requires sufficient depletion layer thickness; for all wavelengths it means that the pixel fill factor has to be close to unity

- $\quad$ High dynamic range: low readout noise in combination with high full well

- (Close to) snapshot imaging: the exposures for all pixels have to start and stop at the same time instant

- Good uniformity, in brightness and darkness, good cosmetics

- Low dark current

- $\quad$ Sufficient radiation hardness

The two detector types we have considered for the TROPOMI UV-NIR channels are CCD and CMOS detectors. CCD's have been used in many science missions as well as our heritage instrument OMI. CMOS detectors have a promise of providing improved radiation hardness, low power dissipation and easy drive electronics.

The difference between $\mathrm{CCD}$ and CMOS detectors is interesting because there are several rather recent claims for good radiation hardness of CMOS detectors (ref 3).

The basic difference between CCD and CMOS in this respect is that the CCD has a charge transfer step which is susceptible to lattice defects from especially high energetic protons. This clearly forms a concern for astronomic missions (e.g. GAIA) but it was never a difficulty in the OMI instrument. This may firstly be explained by the fact that Earth observation instruments relatively see high signals and therefore traps due to dislocations are constantly filled and do not deteriorate the charge transfer process. Another explanation may be that charge transfer degradations are obscured because the spectrograph slit covers typically three detector pixels and this may hide any tailing caused by a less ideal charge transfer.

With respect to dark current, the advantage for CMOS detectors is also not as clear as we originally thought. The issue is that CMOS as well as CCD detectors have diodes for the actual detection of photons and any radiation induced damage should affect the dark current in both detector types in the same way. It may very well be that, due to the fact that CCD's are more mature, they have a lower dark current where increases are more easily seen. Typical example of the difference in maturity is that contrary to CMOS, CCD's use inverted mode operation which significantly reduces dark current. Anyhow, a difference in dark current may be overcome by lowering the detector operating temperature and this is easier in the case of CMOS detectors because of their generally lower dissipation.

There is also an essential advantage for CCD's in the sense that the binning we require can be performed straightforwardly within the detector chip, i.e. before using the output amplifier and thereby adding readout noise. This means that even if a CMOS detector is developed with the same readout noise as a CCD, the advantage for the CCD is still a factor 2 when binning 4 pixels. This difference is not easily overcome with CMOS detectors.

Together with the poorer maturity of CMOS detectors (especially in the UV sensitivity but also e.g. uniformity and cosmetics), we had sufficient arguments to chose CCD detectors in the baseline design for TROPOMI.

For the SWIR we cannot use a CCD and require MCT-CMOS detectors where the MCT band gap is trimmed to about $2.5 \mu \mathrm{m}$ cut-off wavelength. Such detectors are both commercially available and under development. The number of pixels is however smaller than for the UV-NIR $(256 \times 1000)$ and therefore the SWIR optics design has cylindrical optics to allow working with these.

\section{OVERALL INSTRUMENT LAYOUT}

Fig. 4 shows the TROPOMI overall functional diagram. It shows the separate UV-NIR and SWIR modules, each with its telescope, calibration functions and thermal radiator. The UV-NIR and SWIR have a common electronics unit (ICU). The common calibration functions consist of sun diffuser measurements and white light source (WLS). The SWIR has also a spectral line source (SLS), probably in the form of laser diodes. 


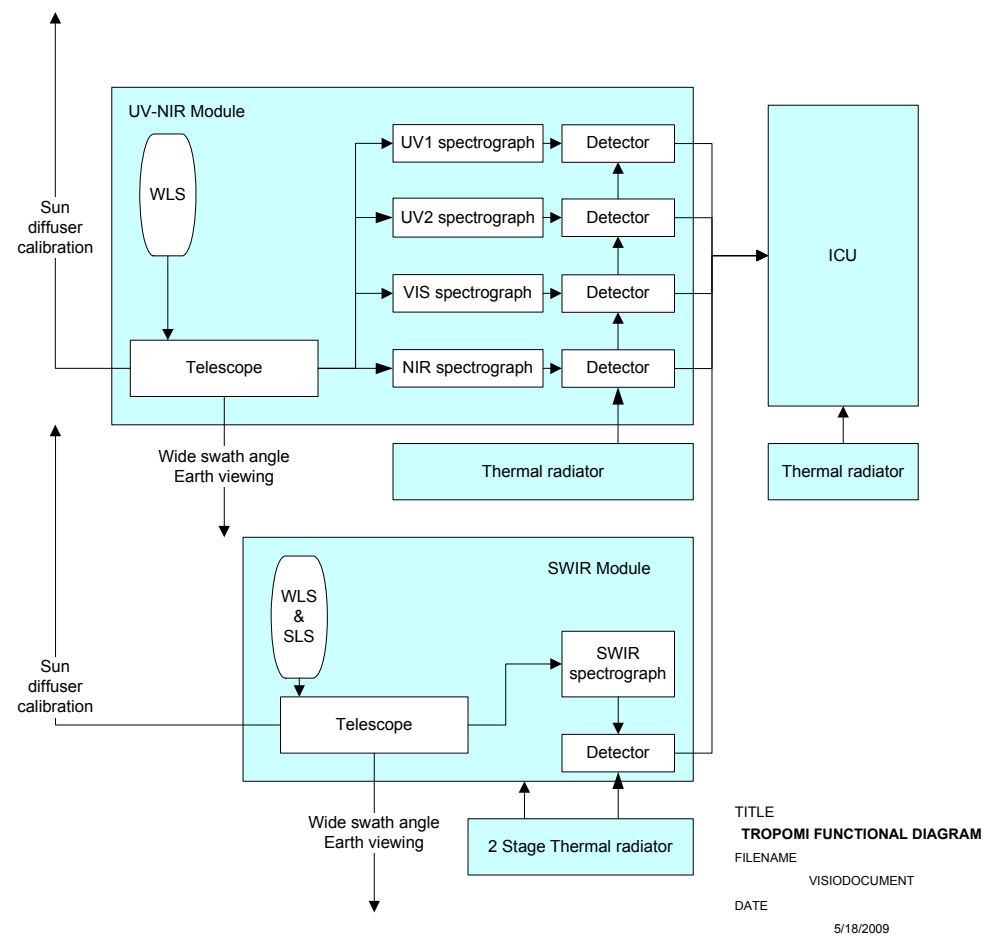

Fig. 4 TROPOMI functional diagram

Fig. 5 shows a provisional mechanical layout with the two modules indicated, each with its detector modules including front-end electronics and calibration units.

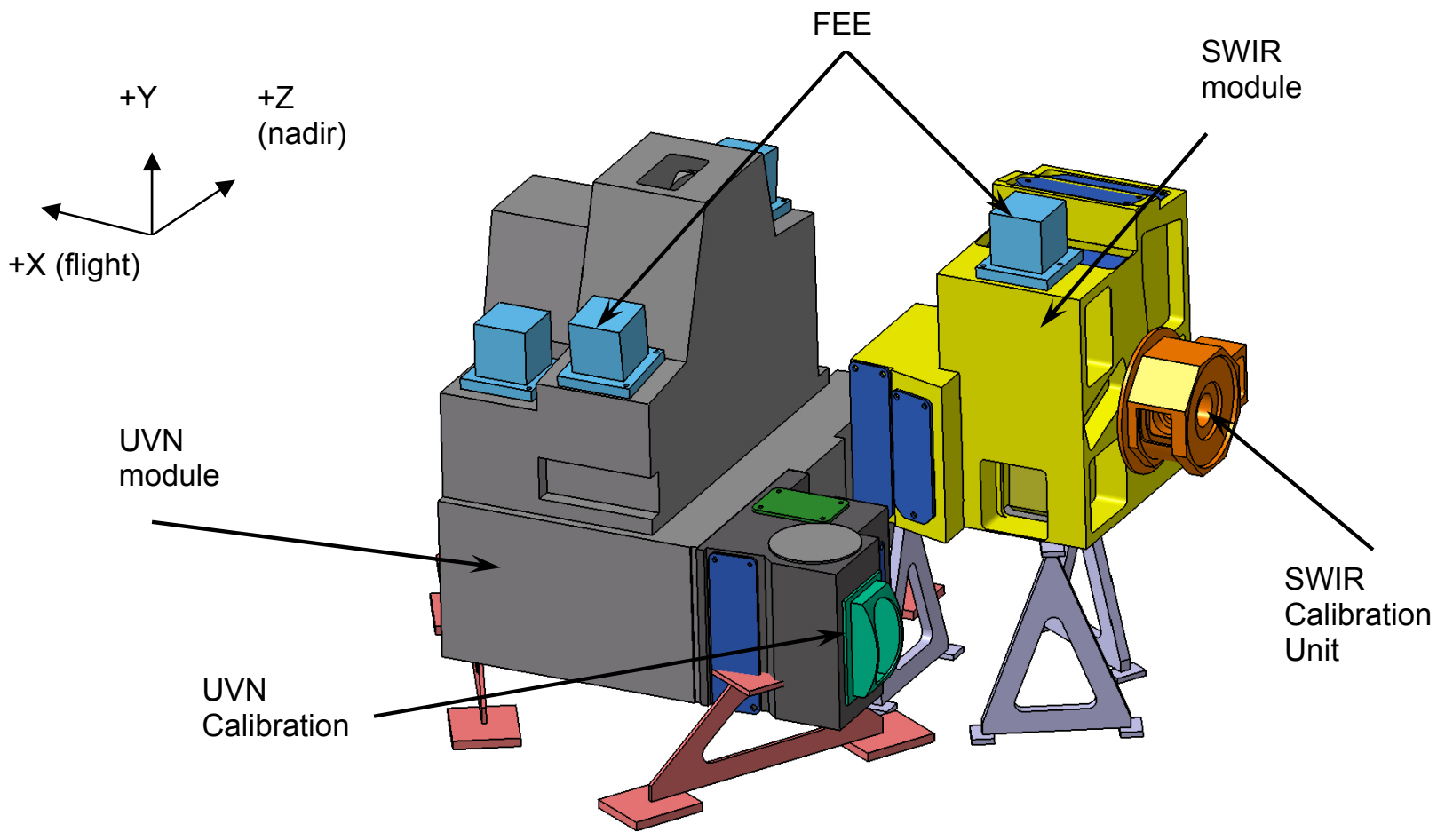

Fig.5 TROPOMI mechanical layout 


\section{POLARIZATION EFFECTS IN LEVEL 2 DATA USING THE TIDE SIMULATOR}

In the light of the TROPOMI development, we have started a grid-based simulation tool TIDE (TROPOMI Integrated Development Environment) to compute Level 2 data product accuracies for the given TROPOMI design. The tool is a sequence of the following process steps.

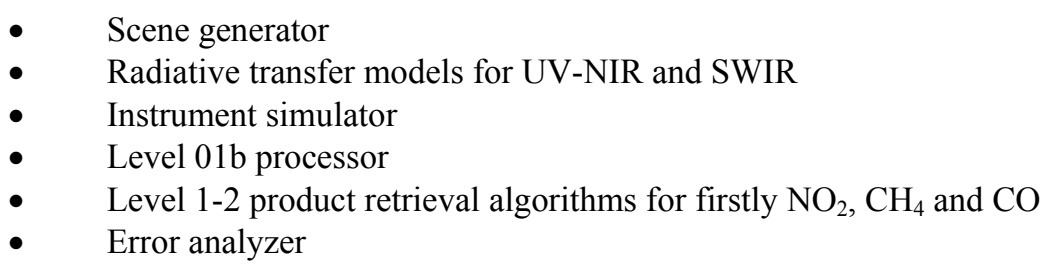

The task of the Scene generator is to define the observed scene, the orbit and to re-grid the scene to the instrument observed ground pixels. The radiative transfer models compute for every scene pixel the top-of-the-atmosphere radiance. The instrument simulator generates Level 0 data and the Level $01 \mathrm{~b}$ converts these into Level $1 \mathrm{~b}$ product files. Level 1-2 product retrieval algorithms compute trace gas total columns. The final step is formed by an Error analyzer that compares the trace gas content in the input and result scenes.

The tool includes science partners for the Radiative transfer models and product retrieval algorithms (KNMI and SRON), instrument development partners (Dutch Space and TNO) for the instrument model and design data and the Level 01b processor and last, but not least a commercial data user partner (BMT ARGOSS) that has generated a large range of realistic test scenes and assimilates the result data into their chemistry transport models.

In a recent test, we have simulated the Level 2 effects from the polarization scrambler in TROPOMI. The performance of this scrambler is a careful balance between a spatial effect, residual polarization and radiometric polarization features. The spatial effect smears the ground pixels somewhat and this is especially critical in view of the good spatial resolution of TROPOMI $(7 \mathrm{~km})$. The other effects degrade the trace gas accuracy and are also unwanted.

The scrambler consists of 4 birefringent quartz wedges which effectively cause the different polarization components to average out. However, the mere presence of the wedges introduces a slight polarization dependence of the viewing angles of the instrument (this is the spatial effect) and incompleteness of the averaging process results in residual polarization and a fractional radiometric residual in the order of a few times $10^{-4}$ (this is what we called the 'features'effect). The radiometric effect is largely because the scrambler is behind the first telescope mirror and therefore does not 'scramble' the polarization effects of this mirror.

The residual and radiometric effects on the $\mathrm{NO}_{2}$ retrieval accuracy are the topic of study and have been included in the TROPOMI instrument simulator. The simulator for this reason uses fully polarized input radiances and has a Mueller matrix description of the telescope, the scrambler, the grating and remaining optics.

The error matrices are shown in Fig.6. They show the difference from two simulation runs: one with the full Mueller matrices and one where the feature-type Mueller elements are zeroed as well as the off-diagonal elements for the first telescope mirror and first scrambler surface. This represents the difference between the scrambler as designed and an idealized scrambler.

The results are shown for two cases (left and right graph in Fig.6), on the left where the Mueller matrices for the central swath angles are used and one on the right where the matrices are used valid for the extreme swath angles ( $\sim 50$ degrees). This represents the case where the satellite overpasses the scene used in the Netherlands and one where the satellite overpass is half the swath width $(\sim 1300 \mathrm{~km})$ from the Netherlands.

The errors range from $5.4 .10^{-5}$ to $7.1 .10^{-4}$ for the central part of the swath and from $-2.7 .10^{-3}$ to $-7.8 .10^{-4}$ for the extreme swath angles. This means that the maximum error is about $0.3 \%$, which is low in relation to other error sources. 

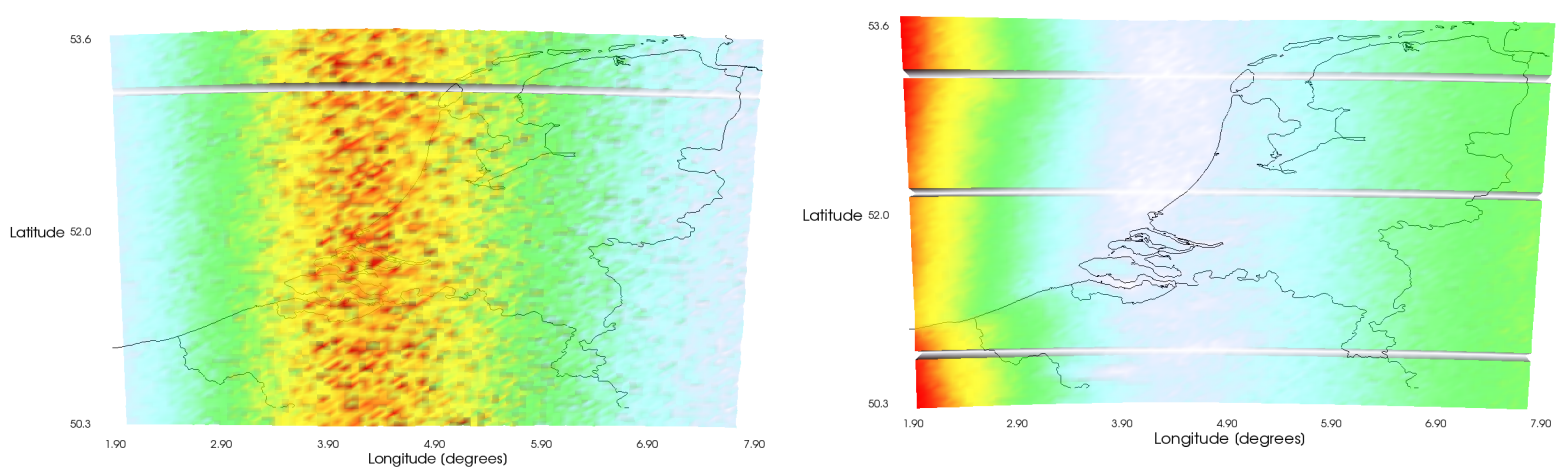

Fig. 6 Error matrices for $\mathrm{NO}_{2}$ retrieval when isolating the polarization effects by the scrambler; the left graph uses the scrambler properties for the central part of the swath and the right for the extreme swath angles; the absolute values of the errors range from 5.4.10 $0^{-5}$ to $7.1 .10^{-4}$ for the left graph and from $-2.7 .10^{-3}$ to $-7.8 \cdot 10^{-4}$ for the right

\section{FURTHER DEVELOPMENT OF THE TIDE SIMULATOR}

We estimate that these results are fairly representative, but a known limitation so far is that the sun angles have been kept constant in these computations. This means that a possible seasonal dependency is not included in the results shown above.

However, the simulator has been improved in the meanwhile and the results from the TIDE simulator are by now fully geo-located. It uses a typical polar orbit and representative ground pixel sizes from TROPOMI. Fig.7 shows an $\mathrm{NO}_{2}$ scene above the Netherlands (it should be clear by now that the authors are from the Netherlands) and a TROPOMI representative observation grid.

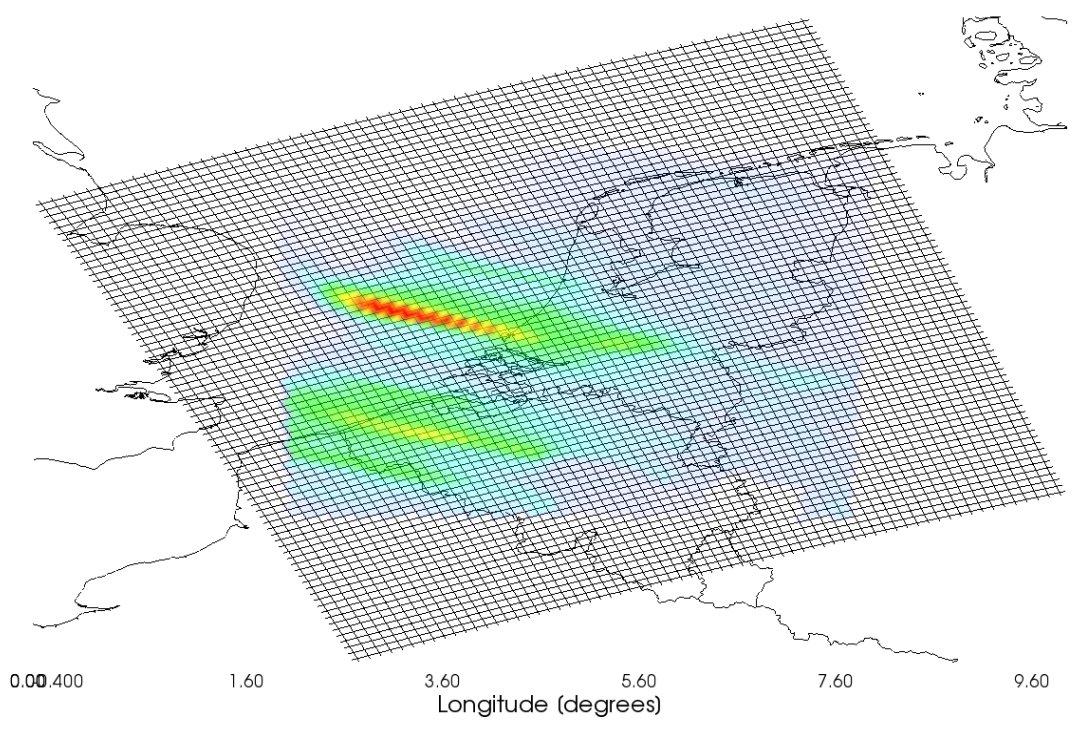

Fig. $7 \mathrm{NO}_{2}$ scene above the Netherlands and a TROPOMI representative $7 \times 7 \mathrm{~km}^{2}$ observation grid 


\section{CONCLUSION}

Having seen the successes of SCIAMACHY and OMI, we expect that TROPOMI will form a further improvement in the fields of air quality observations from space and climate research. It will outperform the older instruments in terms accuracy and localization of trace gas sources and sinks and sensitivity.

However, with its launch in 2014 we are only at the start of a complex programme and there are many challenges to overcome. Following the Dutch national instrument development phases, we have to build an international industrial consortium to realize the instrument and the platform.

Having seen the interest in the programme, we are confident that good partners will be selected to realize this interesting mission.

\section{REFERENCES}

[1] Bovensmann, H., et al., 1999, "SCIAMACHY - mission objectives and measurement modes", J. Atmos. Sci., 56, $127-150$

[2] P.F. Levelt, G.J.H. van den Oord, M.R. Dobber, A. Mälkki, H. Visser, J. de Vries, P. Stammes, J.O.V. Lundell, H. Saari., "The Ozone Monitoring Instrument", IEEE Transactions on Geoscience and Remote Sensing, Vol.44, No.5, May 2006

[3] J. Bogaerts, "Radiation-induced degradation effects in CMOS Active Pixel Sensors and design of a radiation-tolerant image sensor", $\mathrm{PhD}$ thesis, 2002

[4] Y. Bai, S.G.Bernd, J.R. Hosack, M.C. Farris, J.T. Montroy, J. Bajaj ,’Hybrid CMOS Focal Plane Array with Extended UV and NIR Response for Space Applications"; Presented at SPIE's 48 ${ }^{\text {th }}$ Annual Meeting, 3-8 Aug. 2003, San Diego, CA 